\title{
Distribution of serum specific IgE in children with allergic conjunctivitis and analysis of its concomitant allergic diseases
}

\author{
Wenjun Wang", Xiumei Dü, Lu Ye, Xiaoli Wang, Guoyun Zhang \\ Department of Optometry Center, Xi'an People's Hospital (Xi'an Fourth Hospital), Xi'an, China \\ Contributions: (I) Conception and design: W Wang, X Du; (II) Administrative support: L Ye; (III) Provision of study materials or patients: W Wang, \\ X Du, X Wang, G Zhang; (IV) Collection and assembly of data: All authors; (V) Data analysis and interpretation: W Wang, L Ye, X Wang, G Zhang; \\ (VI) Manuscript writing: All authors; (VII) Final approval of manuscript: All authors. \\ \#These authors contributed equally to this work as co-authors. \\ Correspondence to: Wenjun Wang. Department of Optometry Center, Xi'an People's Hospital (Xi'an Fourth Hospital), No. 21, Jiefang Road, Xincheng \\ District, Xi'an, China. Email: wangwjchina@126.com.
}

Background: Allergic conjunctivitis (AC) is predominantly caused by serum specific-IgE (sIgE)-mediated type I allergy. This study aims to analyze the distribution of $\mathrm{sIgE}$ in children with AC, and the concomitant allergic diseases.

Methods: The clinical data from 310 children, diagnosed with AC and admitted to our hospital from January 2017 to January 2019 were retrospectively collected. The children were divided into three groups: infant group ( 2 months to 1 year old, 91 cases), child group (1 to 3 years old, 112 cases), and preschool group (3 to 6 years old, 107 cases). Children in every group were analyzed for positive rates, the number of positive $\mathrm{sIgE}$ types, the distribution of positive inhaling and ingesting allergens and concomitant allergic diseases.

Results: The sIgE positive rate of infant was significantly lower than that of the other two groups, and the number of $18.75 \% \mathrm{sIgE}$ positive species was 1 . The number of sIgE positive species in the child group and preschool group was more than $2(78.30 \%, 71.15 \%)$. The positive rate of sIgE to dust mites, house dust, animal dander, eggs, beef, mutton and mango in the preschool group was significantly higher than the other groups $(\mathrm{P}<0.05)$. The positive rate of $\mathrm{sIgE}$ to milk in infant group was significantly higher than the other two groups $(\mathrm{P}<0.05)$. Children in the preschool group showed the highest incidence of $\mathrm{AC}, \mathrm{AC}+$ allergic rhinitis, $\mathrm{AC}+$ allergic rhinitis + wheezing, while those in the infant group displayed the lowest incidence $(\mathrm{P}<0.05)$. $\mathrm{AC}+$ gastrointestinal allergy, $\mathrm{AC}+$ atopic dermatitis, $\mathrm{AC}+$ gastrointestinal allergy + atopic dermatitis in infant group was significantly higher than the other two groups $(\mathrm{P}<0.05)$. Patients in the child group displayed a significantly higher incidence of $\mathrm{AC}+$ infant wheezing than the other two groups $(\mathrm{P}<0.05)$.

Conclusions: We correlated children's age with the positive rate and gradual increase in types of AC allergens. Concomitant allergic diseases of children with $\mathrm{AC}$ at different ages conform to the natural course of allergic diseases. In clinic, improving the diagnostic efficiency of AC in children, and early interventional treatment will positively contribute to their prognosis, and reduce the risk of other allergic diseases.

Keywords: Allergic conjunctivitis in children (AC in children); serum-specific IgE (sIgE); concomitant allergic disease

Submitted Jul 06, 2020. Accepted for publication Oct 12, 2020.

doi: $10.21037 / \mathrm{tp}-20-216$

View this article at: http://dx.doi.org/10.21037/tp-20-216 


\section{Introduction}

Allergic conjunctivitis (AC) is a conjunctival allergic reaction, caused by frequent contact of conjunctiva with pollen, dust, animal hair and other antigens in the peripheral environment. Recognized as one of the common diseases in ophthalmology and amongst the most frequent noninfection ocular disorders in pediatric patients, AC is mostly caused by IgE-mediated type I allergy. The symptoms appear within 6-12 hours after antigen stimulation, and peaks within 48-72 hours, potentially remaining for several days $(1,2)$. With the development of medical technology, pediatric AC has gradually received clinical attention in recent years. However, due to the difference in children's activity range and life characteristics from adults, little research has been conducted on the distribution of allergens in children (3). Previous literatures have proposed the correlation between allergic rhinitis, asthma and eczema in allergic diseases, so as to prove that asthma and rhinitis belong to joint diseases and can coexist in one patient from various aspects of epidemiology, pathology and anatomy, and also proposed the concept of "one airway, one disease" (4). Relevant studies have reported that in addition to the allergenic constitution and genetic factors, the environment is an important risk factor of allergic diseases, which is closely related to allergic diseases (5). However, there are few reports on the correlation between food and allergens. In order to explore the relationship between the distribution of sIgE in children with $\mathrm{AC}$ and its concomitant allergic diseases such as gastrointestinal allergies, allergic rhinitis, wheezing in children and other allergic diseases, this article reports the findings from a retrospective study, based on the clinic data of AC children (310 cases) admitted to our hospital from 2017 to 2019. The study aims to strengthen the understanding of $\mathrm{AC}$ in children and provide some references for clinical prevention and treatment. We present the following article in accordance with the MDAR reporting checklist (available at http://dx.doi.org/10.21037/ tp-20-216).

\section{Methods}

\section{General information}

The clinical data from 310 cases of children with AC, admitted to our hospital from January 2017 to January 2019 were retrospectively collected. The data comprised of 197 males and 113 females, aged from 2 months to 6 years, with an average age of $2.33 \pm 2.10$ years old. Data from the children were divided into three age groups: 91 cases in infant group, 112 cases in child group, and 107 cases in preschool group. Inclusion criteria: (I) patients meet the diagnostic criteria of AC (6), with clinical manifestations such as redness, itchy eyes, photophobia, tears, conjunctiva papilla and follicles, eyelid edema, color change of bulbar conjunctiva, conjunctival hyperemia, conjunctival edema and other discomfort of eyes; (II) patients with a clinical history related to allergic diseases and a clear history of exposure to defined or undefined allergens, exhibited in a specific environment, climate, and season; (III) diagnosis of gastrointestinal allergies, wheezing, atopic dermatitis, allergic rhinitis and other allergies examined and confirmed by relevant departments; (IV) treatment naïve patients who have never received other treatments after diagnosis; (V) patients with complete clinical and laboratory data; (VI) children's mothers and their legal guardian were informed consent to this study. Exclusion criteria: (I) patients with conjunctivitis due to other allergic reactions; (II) patients diagnosed with other eye diseases; (III) patients who do not meet the above diagnosis criteria.

This study was approved by Xi'an People's Hospital (Xi'an Fourth Hospital) (No. 2019-131). All procedures performed in this study involving human participants were in accordance with the Declaration of Helsinki (as revised in 2013), and informed consent was taken from all the patients.

\section{Examination methods}

A total of $2 \mathrm{~mL}$ of blood was collected intravenously prior to serum separation via centrifugation. Phadia250 automatic in vitro detection system (Phadia, Sweden) was used for the detection of $\operatorname{sIgE}$, and the immune capture method was employed to detect 20 types of sIgE in the serum samples. The allergens were broadly categorized into two types: inhalation allergens including house dust mites, house dust, cockroaches, animal dander, mold combination, mixed grass and plants; and ingestion allergens including milk, eggs, beef, lamb, fish, seafood and mango. Positive results were evaluated as follows: grade $0(<0.35)$ was negative, and positive grades were $1(<0.70), 2(<3.5), 3(<17.5), 4(<50), 5$ $(<100)$, and $6(>100)$.

\section{Observation indicators}

The relevant examination results of the children were collected to analyze the positive rates and number of 
Table 1 Serum sIgE positive rates and the distribution of positive types in the three groups (\%)

\begin{tabular}{|c|c|c|c|c|c|}
\hline Item & Infant & Child & Preschool & $\chi^{2}$ & $\mathrm{P}$ \\
\hline Positive rate (\%) & $80(87.91)$ & $106(94.64)^{\mathrm{a}}$ & $104(97.20)^{\mathrm{a}}$ & 7.371 & 0.025 \\
\hline slgE positive distribution & & & & 9.633 & 0.008 \\
\hline 1 type & $31(38.75)$ & $23(21.70)^{\mathrm{a}}$ & $21(20.19)^{a}$ & & \\
\hline
\end{tabular}

${ }^{\mathrm{a}}$, was compared with the infant group, $\mathrm{P}<0.05$. slgE, specific-lgE.

positive sIgE types across different ages. The distribution of positive sIgE to inhaled and ingested allergens, and other allergic diseases were also examined.

\section{Statistical analysis}

Statistical analysis of the data in this study was performed using SPSS 28.0 software (IBM, USA). The count data was expressed as a percentage or number of cases and analyzed using the $\chi^{2}$ test. Results with $\mathrm{P}<0.05$ was considered statistically significant.

\section{Results}

\section{Distribution of sIgE positive rate and number of positive types in the three groups}

Significant differences were noted in the distribution of sIgE positive rates and the number of positive types in children of the three groups $(\mathrm{P}<0.05)$. The positive rate of children in the infant group was significantly lower than that of the child and preschool group. Only $1 \mathrm{sIgE}$ positive type was identified in infant group (38.75\%), while most of children in the child and preschool groups had more than two positive types of sIgE, counting for $78.30 \%, 71.15 \%$, respectively (Table 1).

\section{Total serum IgE positive rate of children in the three groups}

The total serum IgE level of the three groups was detected, and the results showed that the total positive rate of $\mathrm{IgE}$ was 91.29\% (283/310), which was not statistically significant compared with the sIgE positive rate of $93.55 \%(290 / 310)$ $(\mathrm{P}>0.05)$.

\section{The positive distribution of total IgE and sIgE of inhaled allergens in AC children in the three groups}

Significant differences were noted in the positive distribution of $\operatorname{sIgE}$ to house dust mites, house dust and animal dander in children of the three groups $(\mathrm{P}<0.05)$ The presence of sIgE to house dust mites, house dust and animal dander in the preschool group was significantly higher than the other two groups $(\mathrm{P}<0.05)$, while no significant difference was observed in the positive distribution of $\operatorname{sIgE}$ among cockroaches, mold combinations, mixed grasses, and plants in the three groups $(\mathrm{P}>0.05)$. The details are shown at Table 2 and in Figure 1.

\section{Positive distribution of sIgE to ingestion allergens in children with AC in the three groups}

There were significant differences observed in the positive distribution of sIgE to milk, eggs, beef, mutton, and mango among children in the three groups $(\mathrm{P}<0.05)$. The $\operatorname{sigE}$ positive rate to milk in infant was significantly higher than that of the other two groups $(\mathrm{P}<0.05)$. Children in the preschool group displayed significantly higher sIgE positive rates to eggs, beef, lamb, and mango compared to the other two groups $(\mathrm{P}<0.05)$. However, no statistical difference was noted in the positive distribution of sIgE between fish and seafood in all groups $(\mathrm{P}>0.05)$. The details are shown in Table 3 and Figure 2.

\section{Distribution of concomitant allergic diseases in the three groups}

There were significant differences in the distribution of concomitant allergic diseases in children of the three groups $(\mathrm{P}<0.05)$. Children in the preschool group displayed 
Table 2 The sIgE positive distribution of inhaled allergens of children with AC in the three groups

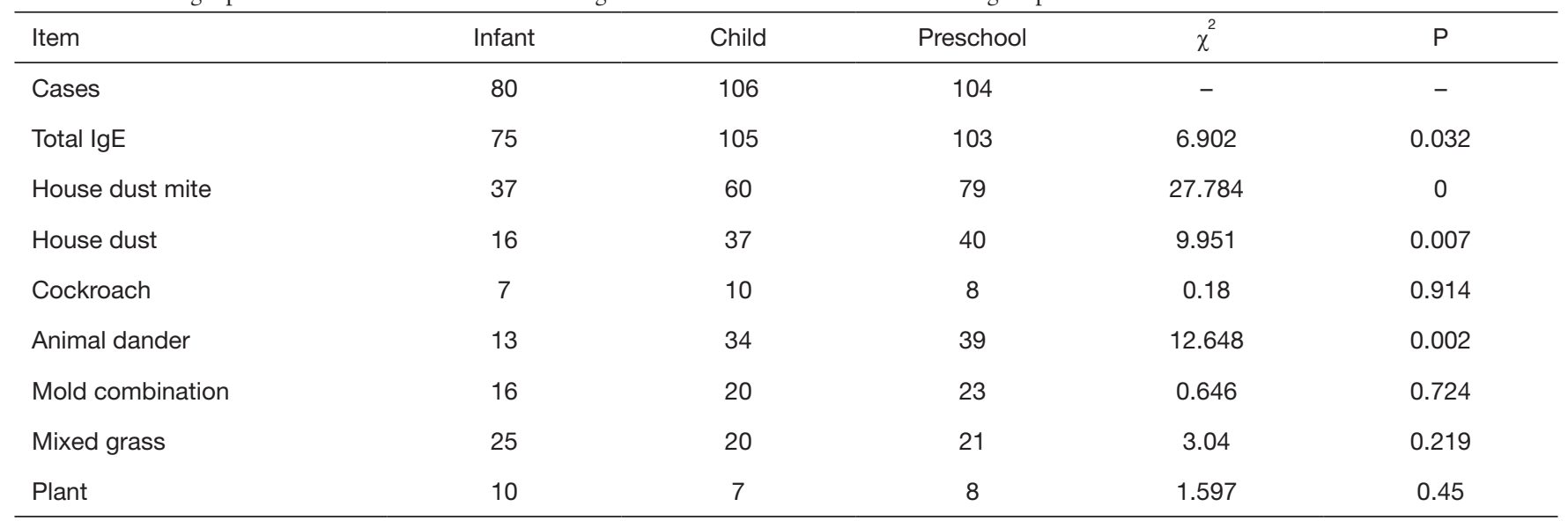

slgE, specific-lgE; AC, allergic conjunctivitis.

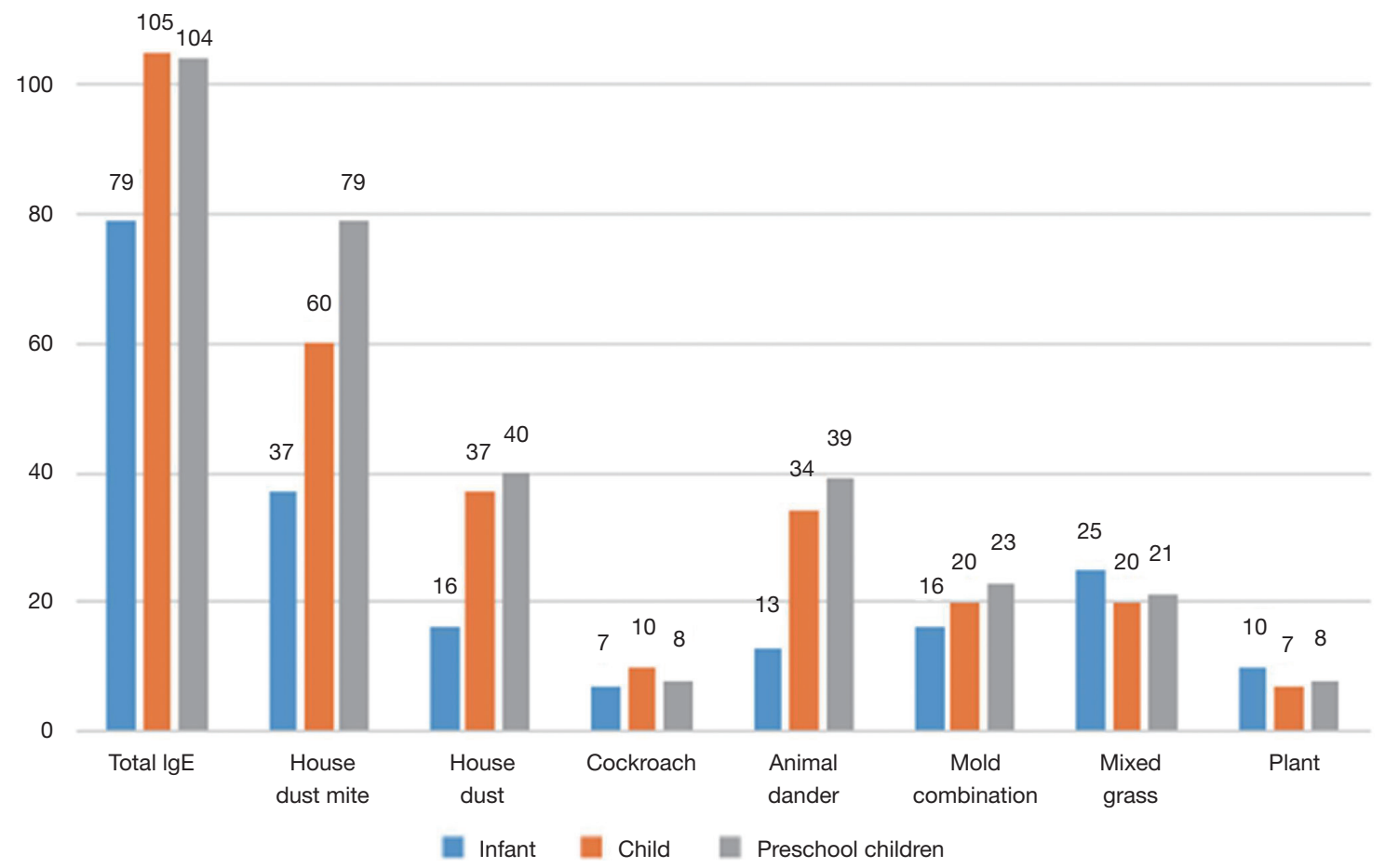

Figure 1 The positive distribution of inhaled allergen total $\operatorname{IgE}$ and $\operatorname{sgE}$ in the three groups.

the highest incidence of $\mathrm{AC}, \mathrm{AC}+$ allergic rhinitis, and $\mathrm{AC}+$ allergic rhinitis + infant wheezing, while children in the infant group showed the lowest incidence of the same allergic diseases $(\mathrm{P}<0.05)$. Children in the infant group had a higher incidence of $\mathrm{AC}+$ gastrointestinal allergy, $\mathrm{AC}+$ atopic dermatitis and $\mathrm{AC}+$ gastrointestinal allergy + atopic dermatitis than the other groups $(\mathrm{P}<0.05)$. Furthermore, children in the child group had a significantly higher incidence of $\mathrm{AC}+3$ or more allergic diseases, and $\mathrm{AC}+$ infants wheezing than the other two groups $(\mathrm{P}<0.05)$. The details are shown in Table 4 and Figure 3. 
Table 3 The sIgE positive distribution of injection allergens in children of the three groups

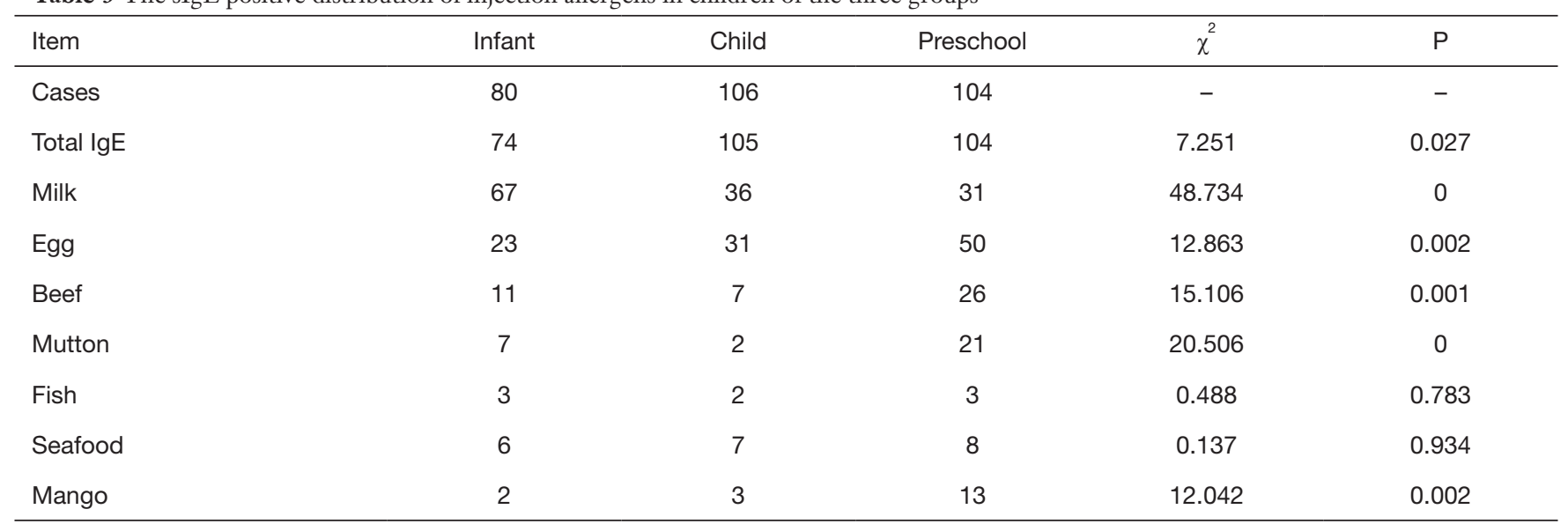

slgE, specific-lgE.

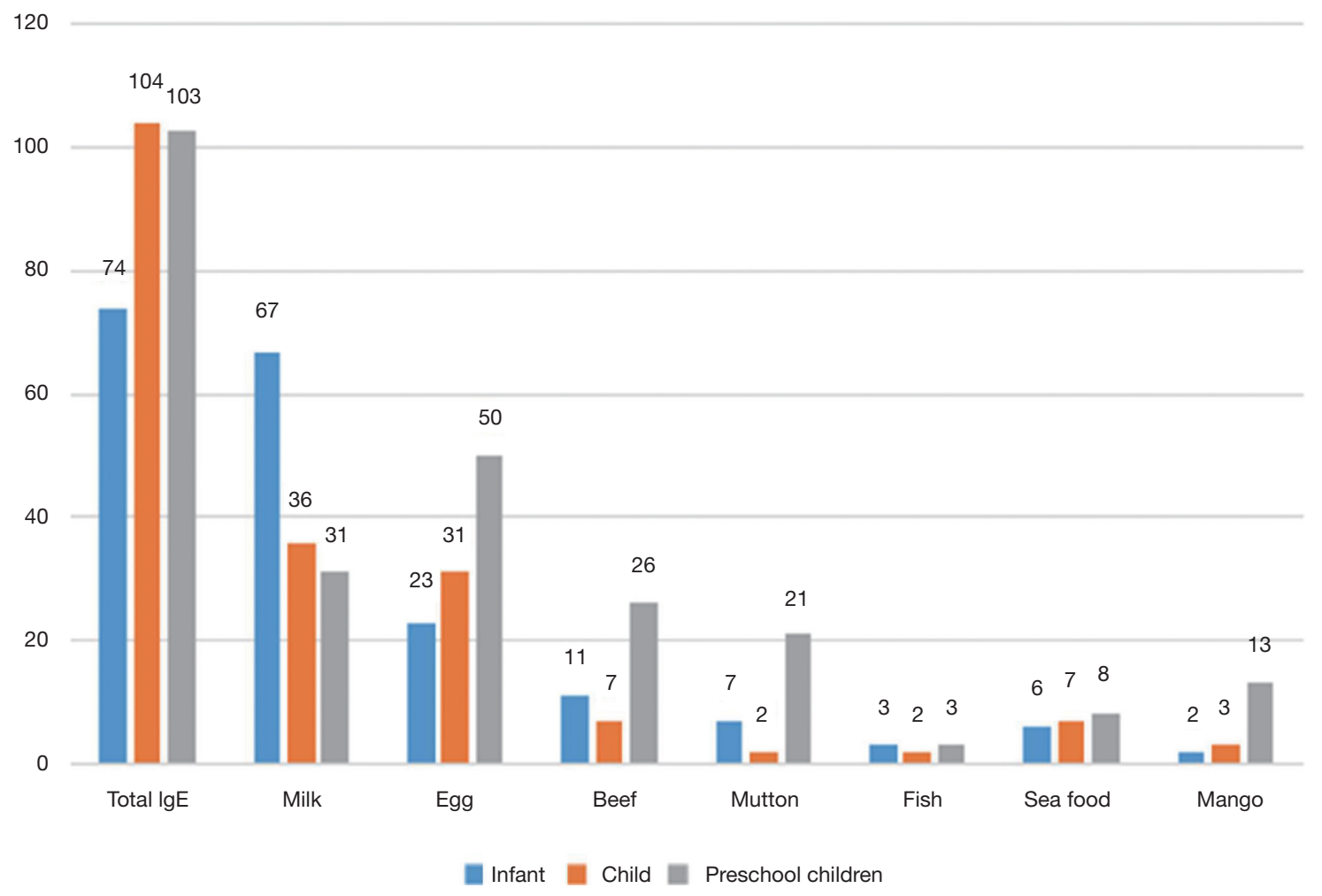

Figure 2 The positive distribution of total $\operatorname{IgE}$ and sIgE of allergen ingestion in children with AC in three groups. AC, allergic conjunctivitis. 
Table 4 Distribution of children with other allergic diseases in the three groups

\begin{tabular}{lcccc}
\hline Item & Infant & Child & Preschool & $\chi^{2}$ \\
\hline Cases & 80 & 106 & 104 & - \\
AC alone & 7 & 18 & 31 & 15.516 \\
AC + allergic rhinitis & 2 & 19 & 33 & 28.073 \\
AC + gastrointestinal allergy & 20 & 9 & 3 & 20.522 \\
AC + atopic dermatitis & 22 & 10 & 7 & 16.031 \\
AC + infant wheeze & 5 & 18 & 9 & 6.127 \\
AC + gastrointestinal allergy + atopic dermatitis & 24 & 4 & 1 & 0 \\
AC + allergic rhinitis + infant wheeze & 1 & 14 & 22 & 0 \\
AC + 3 or more allergic diseases & 3 & 20 & 10 & 17.035 \\
\hline
\end{tabular}

slgE, specific-lgE; AC, allergic conjunctivitis.

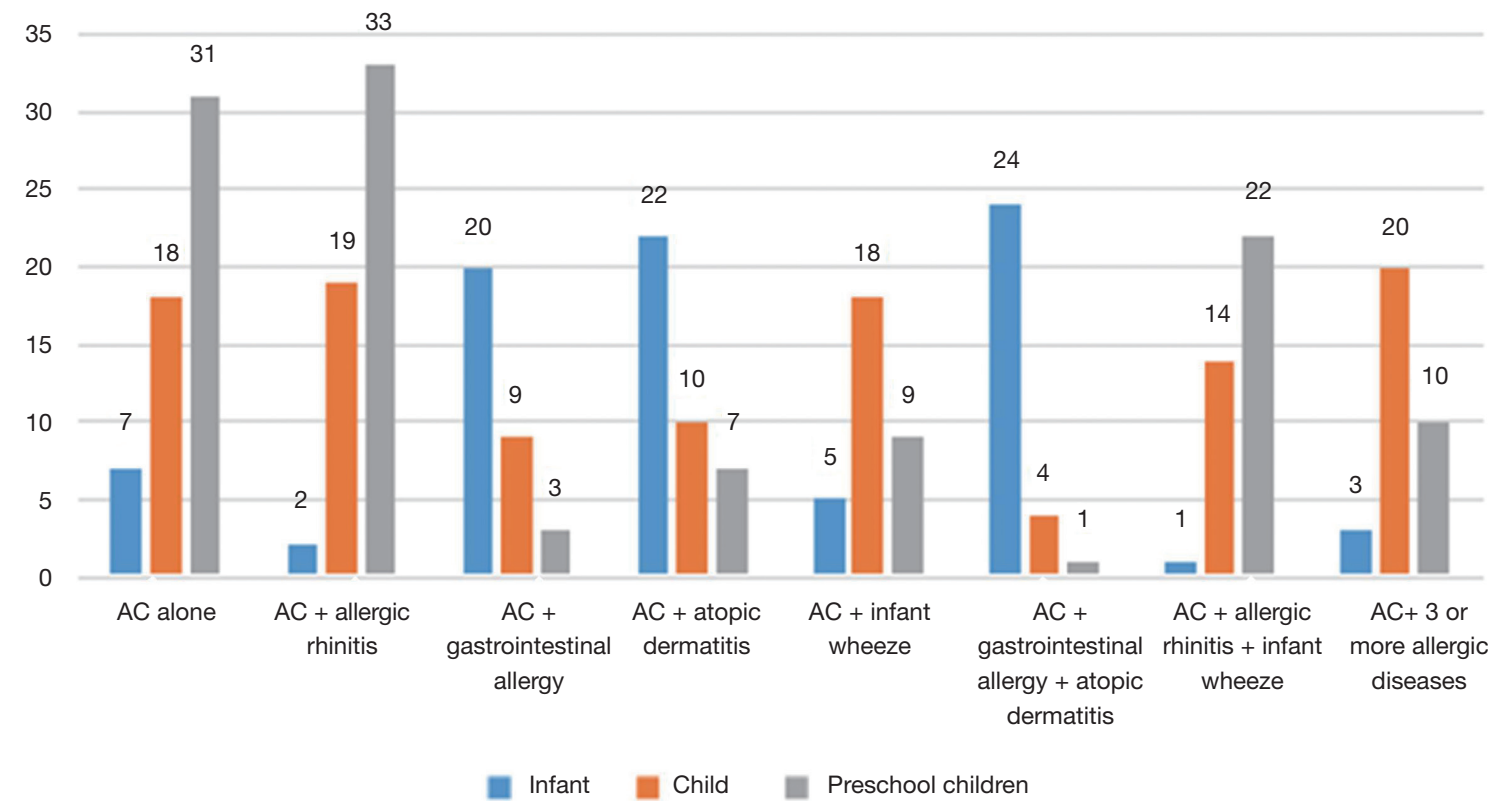

Figure 3 Distribution of other allergic diseases in children of different groups.

\section{Discussion}

Some scholars have pointed out that the eye binding membrane is rich in blood vessels and exposed to external antigens, which can easily stimulate allergic symptoms (7). Allergens are affected by house dust, mites, fungi and other factors in different seasons, and there are many related studies on the increased positive expression of allergens. Therefore, in order to seek new ideas for the treatment of children with AC, this study focus on exploring the relationship between allergens and food, which might provide theoretical guidance for the clinical treatment of children with AC.

In this study, serum examination led to the detection of $290 \mathrm{sIgE}$ positive cases in 310 children diagnosed with AC, with a positive rate of $93.54 \%$. Among them, patients in the infant group had the lowest sIgE positive rate, and 
predominantly exhibited one type of allergen (38.75\%). In addition, children in the child and preschool groups displayed a higher $\mathrm{sIgE}$ positive rate with over two types of allergens $(78.30 \%, 71.15 \%)$. Subsequent analysis of inhalation and ingestion allergens identified house dust mites, house dust, milk and eggs as the main allergens, suggesting a positive correlation between age, $\operatorname{sIgE}$ positive rate and allergen types. Referring to the analysis in previous literatures, we guess this phenomenon might be attributed to a diversifying diet as the child ages, along with increases in their exposure to allergens from outdoor activities $(7,8)$. In the infant group, the positive rate of house dust mites among inhaled allergens in children was much higher compared to other allergens except milk. In contrast to our findings, the majority of previous studies have reported that these allergic symptoms are almost food-related (9). This may be because AC is predominantly related to abnormal inflammatory symptoms of the conjunctiva, induced by direct stimulation. This result also suggests that house dust mites are an important key factor in AC induction, highlighting the significance of maintaining a clean environment for children in the infant group (10). Through the study of a large number of preschool children, it has been found that the probability of inhaled allergens increased significantly with age in children (11). This was further supported by our findings, where the $\operatorname{sgE}$ positive rate of house dust mites in preschool children was the highest among all groups. In addition to house dust mites, the $\operatorname{sgE}$ positive rates of house dust and animal dander were also increased. Additionally, allergens such as mold allergies, mixed grass, and cockroach are also noteworthy to pay more attention. The results in this study indicated that more focus is required on the control of inhaled allergens, particularly with indoor allergens. Reducing the amount of dust mites can effectively reduce the incidence of allergy by maintaining a daily internal environment that is dry, well ventilated, and with a high level of hygiene $(12,13)$.

Distribution analysis of ingesting allergens revealed milk and eggs as the foods which elicited the strongest response, highlighting the need for more focus on investigations into the clinical role of these food allergens in AC (14). Results in this study showed that the positive rate of sIgE to milk was highest in infant group, which gradually decreased with the increase of age. This suggested that their milk tolerance gradually increased with age, which emphasizes the importance of breastfeeding of infants, to effectively prevent milk-induced allergies. In addition, the positive rates of sIgE to eggs, beef, lamb, and mango were mostly found in preschool children, which may be related to the diversity of their diets with the increase in age.

"Allergy process" is a concept proposed by Fouchard in 1973. Generally, atopic dermatitis occurs during infancy, which is predominantly food-related and most commonly induced by milk and eggs. The incidence of wheezing in patients during preschool to school age is particularly high with allergic rhinitis (15). Allergic diseases in children develop dynamically, and $\mathrm{AC}$, as one of these diseases, is often accompanied with various allergic diseases at different ages. In this study, children in the infant group often had AC with gastrointestinal allergies and atopic dermatitis. However, children in the child group predominantly displayed AC with 3 or more allergic diseases, and children in the preschool group were often accompanied with allergic rhinitis. This phenomenon is consistent with the above concept of the "allergic process". In addition, the current literature suggests that for young children with allergic diseases, the probability of multiple allergic diseases will increase significantly with age (16). Therefore, it is critical that the family medical history is assessed in a timely manner in order to understand the morbidity characteristics, genetic conditions, and associated diseases of the individual child. Subsequently, the relevant laboratory examinations can be performed for a comprehensive and detailed assessment of the child's condition, which was conducive to the choice of following treatment. The importance of monitoring for the positive rate of agerelated $\operatorname{sIgE}$ dynamically has been proposed due to the developmental stage of the immune function in infants (17). Therefore, combined with the results in this study, the $\operatorname{sg} \mathrm{E}$ detection of inhalation and ingestion allergens is required for infants, to potentially reduce the early or continuous exposure of these allergens. Some studies have pointed out that the positive rates of total $\mathrm{IgE}$ of ingested allergens and inhaled allergens are higher than that of $\operatorname{sgE}$, but there is little difference in this study, which might be related to the small sample size of this study. Therefore, the relationship between $\mathrm{AC}$ and the age, environment and allergic diseases of children still needs to be further studied (18).

In conclusion, the allergen positive rate and types of AC gradually increased with age. Additionally, the allergic diseases associated with $\mathrm{AC}$ in children at different ages conformed to the natural course of allergic diseases. It demonstrates a substantial contribution to the prognosis of children by improving their diagnosis efficiency of AC 
clinically. This would facilitate the implementation of early clinical intervention, which could reduce the risk of additional allergic diseases.

\section{Acknowledgments}

Funding: None.

\section{Footnote}

Reporting Checklist: The authors have completed the MDAR reporting checklist. Available at http://dx.doi.org/10.21037/ tp-20-216

Data Sharing Statement: Available at http://dx.doi. org/10.21037/tp-20-216

Conflicts of Interest: All authors have completed the ICMJE uniform disclosure form (available at http://dx.doi. org/10.21037/tp-20-216). The authors have no conflicts of interest to declare.

Ethical Statement: The authors are accountable for all aspects of the work in ensuring that questions related to the accuracy or integrity of any part of the work are appropriately investigated and resolved. This study was approved by Xi'an People's Hospital (Xi'an Fourth Hospital) (No. 2019-131). All procedures performed in this study involving human participants were in accordance with the Declaration of Helsinki (as revised in 2013), and informed consent was taken from all the patients.

Open Access Statement: This is an Open Access article distributed in accordance with the Creative Commons Attribution-NonCommercial-NoDerivs 4.0 International License (CC BY-NC-ND 4.0), which permits the noncommercial replication and distribution of the article with the strict proviso that no changes or edits are made and the original work is properly cited (including links to both the formal publication through the relevant DOI and the license). See: https://creativecommons.org/licenses/by-nc-nd/4.0/.

\section{References}

1. Elieh Ali Komi D, Rambasek T, Bielory L. Clinical implications of mast cell involvement in allergic conjunctivitis. Allergy 2018;73:528-39.

2. Lee HJ, Kim BM, Shin S, et al. Superoxide dismutase 3 attenuates experimental $\mathrm{Th} 2$-driven allergic conjunctivitis. Clin Immunol 2017;176:49-54.

3. Zhong JY, Lee YC, Hsieh CJ, et al. Association between Dry Eye Disease, Air pollution and weather changes in Taiwan. Int J Environ Res Public Health 2018;15:2269.

4. Kuo CH, Collins AM, Boettner DR, et al. Role of CCL7 in Type I hypersensitivity reactions in murine experimental allergic conjunctivitis. J Immunol 2017;198:645-56.

5. Liu J, Huang S, Li F, et al. Sympathetic nerves positively regulate eosinophil-driven allergic conjunctivitis via a1-Adrenergic receptor signaling. Am J Pathol 2020;190:1298-308.

6. Xu C, He X, Liu W, et al. An inhibitor peptide of tolllike receptor 2 shows therapeutic potential for allergic conjunctivitis. Int Immunopharmacol 2017;46:9-15.

7. Ren N, Sun R, Xia K, et al. DNA-based hybrid hydrogels sustain water-insoluble ophthalmic therapeutic delivery against allergic conjunctivitis. ACS Appl Mater Interfaces 2019;11:26704-10.

8. Fodor LE, Gézsi A, Gál Z, et al. Variation in the TEK gene is not associated with asthma but with allergic conjunctivitis. Int J Immunogenet 2018;45:102-8.

9. Li H, Wang W, Xu X, et al. Preparation and characterization of novel pimecrolimus nanoparticles for therapy of allergic conjunctivitis. J Biomed Nanotechnol 2017;13:1106-14.

10. Wei CC, Lin CL, Shen TC, et al. Children with allergic diseases have an increased subsequent risk of migraine upon reaching school age. J Investig Med 2018;66:1064-8.

11. Kwon JY, Lee HS, Joo CK. TRPV1 antagonist suppresses allergic conjunctivitis in a murine model. Ocul Immunol Inflamm 2018;26:440-8.

12. Gerstlauer M, Szepfalusi Z, Golden D, et al. Real-life safety of 5-grass pollen tablet in 5-to-9-year-old children with allergic rhinoconjunctivitis. Ann Allergy Asthma Immunol 2019;123:70-80.

13. Leonardi A, Capobianco D, Benedetti N, et al. Efficacy and tolerability of ketotifen in the treatment of seasonal allergic conjunctivitis: Comparison between ketotifen $0.025 \%$ and $0.05 \%$ eye drops. Ocul Immunol Inflamm 2019;27:1352-6.

14. Sacchetti M, Segatto M, Bruscolini A, et al. Changes of NGF pathway in allergic rhinoconjunctivitis: A conjunctival allergen challenge study. Allergy 2019;74:605-7.

15. Carlsten C, Dimich-Ward H, Ferguson A, et al. Atopic dermatitis in a high-risk cohort: natural history, associated allergic outcomes, and risk factors. Ann Allergy Asthma 
Immunol 2013;110:24-8.

16. Fukuda K, Ishida W, Wakasa Y, et al. Oral Immunotherapy for Allergic Conjunctivitis Using Transgenic Rice Expressing Hypoallergenic Antigens. Cornea 2018;37 Suppl 1:S67-S73.

17. Novikova V, Anichkov N, Nevskaia E, et al. GP178 Morphological features of chronic esophagitis in children with concomitant allergic diseases are interrelated with the level of cytokines and neuropeptides. Arch Dis Child 2019;104:A102-A103.

18. Ren N, Sun R, Xia K, et al. DNA-Based Hybrid Hydrogels Sustain Water-Insoluble Ophthalmic Therapeutic Delivery against Allergic Conjunctivitis. ACS Appl Mater Interfaces 2019;11:26704-10.

(English Language Editor: J. Gray)

Cite this article as: Wang W, Du X, Ye L, Wang X, Zhang G. Distribution of serum specific IgE in children with allergic conjunctivitis and analysis of its concomitant allergic diseases. Transl Pediatr 2020;9(5):636-644. doi: 10.21037/tp-20-216 